Recepción: 06 / 05 / 2018

Aceptación: 20 / 07 / 2018

Publicación: 15 / 09 / 2018

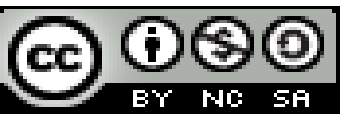

Ciencias económicas y empresariales

Artículo de investigación

\title{
La fabricación de cajas de cartón con material reciclado a través del marketing ecológico a nivel empresarial
}
The manufacture of carton boxes with recycled material through ecological marketing at the business level

\section{A fabricação de caixas de cartonagem com material reciclado através do marketing ecológico a nível de negócio}

\author{
Catalina Falquez-Arce ${ }^{\mathrm{I}}$ \\ catalina.falqueza@ug.edu.ec \\ Frida M. Bohórquez-Suárez II \\ frida.bohorquez@ug.edu.ec \\ Carolina R. Galarza-Menoscal ${ }^{\mathrm{III}}$ \\ carogalarza_94@hotmail.com \\ Brigitte C. Holguin-Tobar ${ }^{\text {IV }}$ \\ gigi_100594@hotmail.com
}

Correspondencia:catalina.falqueza@ug.edu.ec

\footnotetext{
II Ingeniera Comercial, Docente de la Universidad de Guayaquil, Guayaquil, Ecuador.

II Magister en Administración de Empresas, Licenciada en Mercadotecnia, Docente de la Universidad de Guayaquil, Guayaquil, Ecuador.

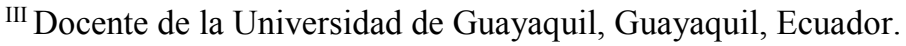

IV Docente de la Universidad de Guayaquil, Guayaquil, Ecuador.
} 


\title{
Resumen
}

Las sociedades han adquirido conciencia ecológica sobre el medioambiente. La utilización de productos fabricados con material reciclado ha ido en auge, aunque todavía se necesita más impulso en esa sociedad altamente consumidora. Es aquí donde entra en juego el uso del marketing ecológico como factor para resaltar aún más los beneficios, no solo del ecosistema, sino propios del producto ecológico para que tenga la aceptación de la sociedad y derribar los paradigmas de mercado existentes. Esta investigación tiene como objetivo analizar la fabricación de cajas de cartón con material reciclado a través del marketing ecológico. La metodología utilizada se basó en investigación no experimental, de campo documental y descriptiva exploratoria. Como conclusión se tiene que los productos ecológicos que provienen del reciclado tienen buenas propiedades los cuales se pueden comparar con los productos fabricados de material virgen haciendo disminuir el impacto ambiental y aumentar el bienestar en el ecosistema.

Palabras claves: reciclado; marketing ecológico; producto ecológico; ecosistema.

\begin{abstract}
The societies have acquired environmental awareness about the environment. The use of products made from recycled material has been booming, although there is still a need for more momentum in this highly consumer society. This is where the use of ecological marketing comes into play as a factor to highlight even more the benefits, not only of the ecosystem, but also of the ecological product so that it has the acceptance of society and tear down the existing market paradigms. The objective of this research is to analyze the manufacture of cardboard boxes with recycled material through ecological marketing. The methodology used was based on nonexperimental research, documentary field and descriptive exploratory. As a conclusion, the ecological products that come from recycling have good properties which can be compared with the products made of virgin material, reducing the environmental impact and increasing the wellbeing in the ecosystem.
\end{abstract}

Keywords: recycled; ecological marketing; organic product; ecosystem. 


\section{Resumo}

As sociedades adquiriram consciência ambiental sobre o meio ambiente. $\mathrm{O}$ uso de produtos feitos a partir de material reciclado tem crescido, embora ainda haja necessidade de mais dinamismo nesta sociedade altamente consumista. É aqui que entra em cena o uso do marketing ecológico para destacar ainda mais os benefícios, não só do ecossistema, mas também do produto ecológico, para que ele tenha a aceitação da sociedade e destrua os paradigmas existentes no mercado. O objetivo desta pesquisa é analisar a fabricação de caixas de papelão com material reciclado através do marketing ecológico. A metodologia utilizada baseou-se em pesquisa não experimental, campo documental e exploratório descritivo. Como conclusão, os produtos ecológicos provenientes da reciclagem apresentam boas propriedades que podem ser comparadas com os produtos feitos de material virgem, reduzindo o impacto ambiental e aumentando o bemestar no ecossistema.

Palavras chave: reciclado; marketing ecológico; produto ecológico; ecossistema.

\section{Introducción}

El desarrollo de las sociedades ha ido de la mano del avance tecnológico con alto impacto en nuestra biodiversidad. El mundo ha comprendido que para poder avanzar en su desarrollo y poder garantizar las necesidades de una sociedad hambrienta de consumo es necesario hacerlo a través de una conciencia ecológica.

Se ha comprendido que estos avances deben hacerse desde el respeto por el medio ambiente ya que la producción ha hecho estragos sobre el planeta tierra produciendo el calentamiento global que sufrimos hoy en día.

Este cambio climático ha producido alteraciones de toda índole manifestando respuestas que perjudican nuestro planeta y el desarrollo de nuestras sociedades. Es por ello que se empieza a producir cambios en la conciencia del ciudadano de cómo desarrollar sin tanto impacto ambiental y como reutilizar productos que ya cumplieron su vida útil. Es aquí donde entra el concepto de reciclaje.

Para poder comprender este nuevo termino es necesario introducir, en todos los niveles de la sociedad, una educación ambiental. Esta educación ambiental debe ir inculcada en todas las 
materias de escolaridad, tanto en educación primaria hasta la educación superior, produciendo en los ciudadanos a futuro un cambio en la conciencia. Si se empieza a edad temprana entonces habrá una mayor sensibilidad hacia los problemas ambientales y posiblemente, producirá un cambio en su comportamiento, por ejemplo, para apoyar el reciclaje (Breiting, 1997).

Indudablemente la educación ambiental producirá cambios significativos en la conducta del individuo, llamándose conducta ambiental. Esta conducta ambiental se ve afectada por variables del entorno de cada individuo como lo son la influencia de la situación física del entorno de la conducta ambiental, las normas sociales, los modelos sociales, los sentimientos y creencias de moralidad asociadas a la conducta ambiental (Corraliza \& Martín, 2000).

Esta educación ambiental que influye directamente en la conducta del individuo debe vencer varios paradigmas sociales. La educación ambiental es saber cultivarse y cultivar a los semejantes $y$, cultivar es asentar la cultura en conceptos profundos y firmes intentando vencer o por lo menos minimizar la existencia y uso de los preconceptos que tienden a sesgar o tergiversar el conocimiento científico (Lara, 2008).

Entonces, es necesario definir el termino reciclado para que sea catalizador en la nueva cultura del individuo. El reciclaje es un proceso simple que sufre un material o producto para ser reincorporado a un ciclo de producción o consumo, ya sea éste el mismo en que fue generado u otro diferente (Henao, 2013).

Sin duda alguna, la utilización del reciclado en la vida cotidiana de la sociedad ha aumentado en los últimos años. Los ciudadanos exigen a las empresas poner en práctica el reciclado y sobre todo implementar la reutilización de algunos de sus productos. Es por ello, que las empresas de generación de tecnología deben diseñar nuevos empaques innovadores, con el fin de crear una cultura de reutilización más que de reciclaje (Chacón, Pacheco, Cendejas, \& Ortega, 2016).

Estas propuestas, generadas por la misma sociedad, es en consecuencia del gran impacto ambiental que se generan por los residuos sólidos. El fuerte impacto ambiental ha generado una campaña de sensibilización que tiene un doble objetivo, la disminución de la contaminación en general y la del consumo de energía (Castro, 2009). 
Uno de los mayores impactos ambientales es la contaminación de papel y cartón, y sobretodo un impacto en la conciencia ambiental del proceso de fabricación de los mismos. Sin embargo, las empresas buscan excusas para la fabricación de papel reciclado considerando que el recuperador se enfrenta a un balance negativo entre el coste de recogida y tratamiento del papel recuperado, por una parte, y la venta al fabricante de papel por otra (Chas \& Lorenzo, 1997).

Por supuesto, existe un costo de producción, pero este precio es mínimo a la gran contribución social, ambiental y global que se debe hacer para el planeta. Existen ventajas para usar papel reciclado. Para fabricar papel a partir de la celulosa virgen se requiere madera, agua y energía; para obtener la misma cantidad con papel usado recuperado se necesita 100 veces menos cantidad de agua y una tercera parte de energía (Agular, 2004). El proceso de producción de papel o cartón reciclado se puede visualizar en la Fig. 1.

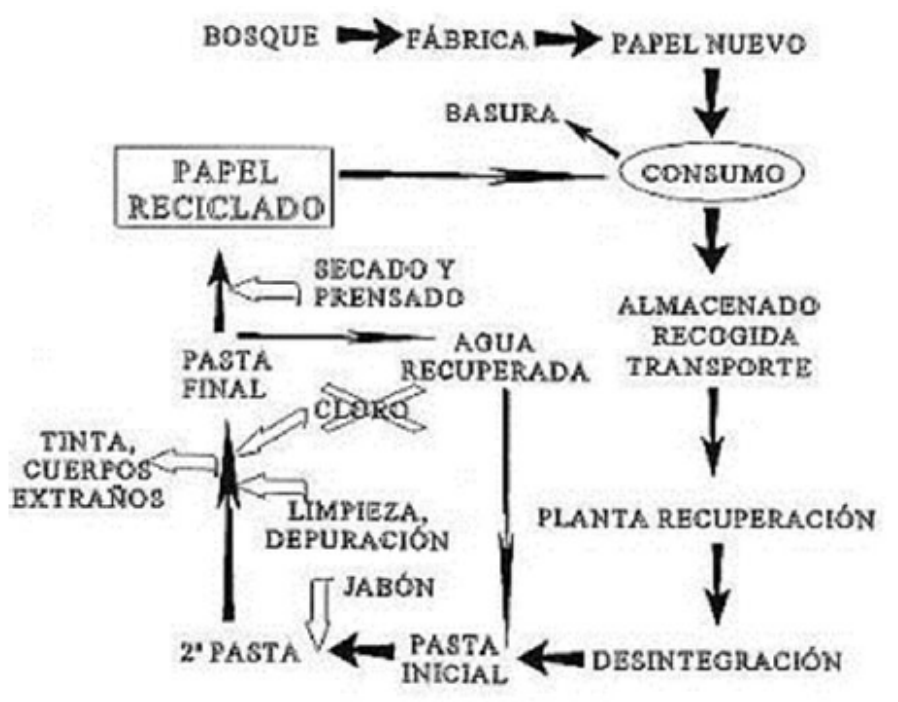

Fig 1. Esquema del proceso de fabricación del papel reciclado. Fuente: (Cadme \& Miranda, 2012)

Existen muchos productos que pueden ser derivados de material reciclado como el papel o cartón. Uno de ellos es la elaboración de cuadernos de apuntes. La gran mayoría utiliza estos cuadernos, sobre todo los estudiantes que buscan en el producto la calidad, el tamaño y el diseño de la cubierta a la hora de elegir un encuadernado (CIFUENTES, 2013). 
También el cartón reciclado es usado para la construcción en obras civiles. En el mercado internacional el uso de papel y cartón va dirigido en su mayoría a aislar acústica y térmicamente las edificaciones debido a propiedades que avalan este uso (Espinal, 2016).

Sin lugar a dudas, esta concientización de algunos factores de la sociedad ha generado esta diversidad de productos provenientes del reciclado, pero aún hace falta más conciencia de todos los ciudadanos. Esto viene por la falta de una comunicación efectiva por parte de los entes gubernamentales como del sector productivo. Es aquí donde hace falta un buen marketing. Esto se puede observar en la Fig. 2 que corresponde a un árbol de problemas para entrar en el mercado y por ende en la conciencia de la sociedad.

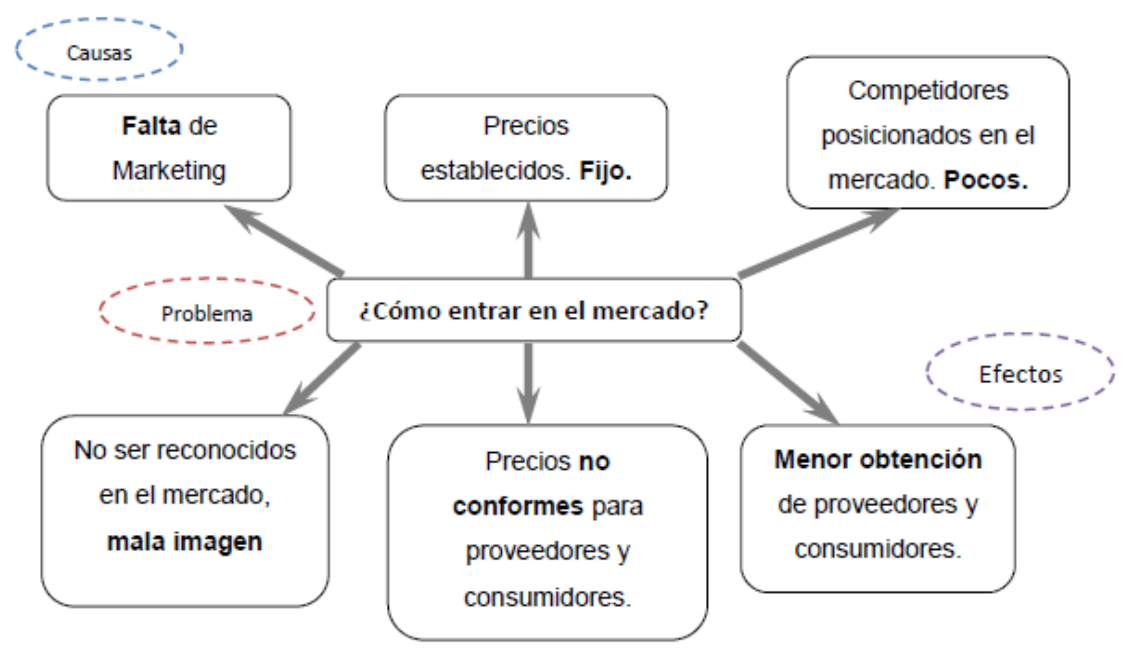

Fig 2. Árbol de problemas para entrar al mercado. Fuente: (Cadme \& Miranda, 2012).

Es por ello que debe existir la necesidad de poder comprender el termino de marketing. Este concepto está vinculado a necesidades, deseos y demandas; producto; valor, satisfacción y emoción; intercambio, transacción y relación; mercado; gestión de marketing (Monferrer, 2013). En la Fig. 3 se puede observar estas características de marketing.

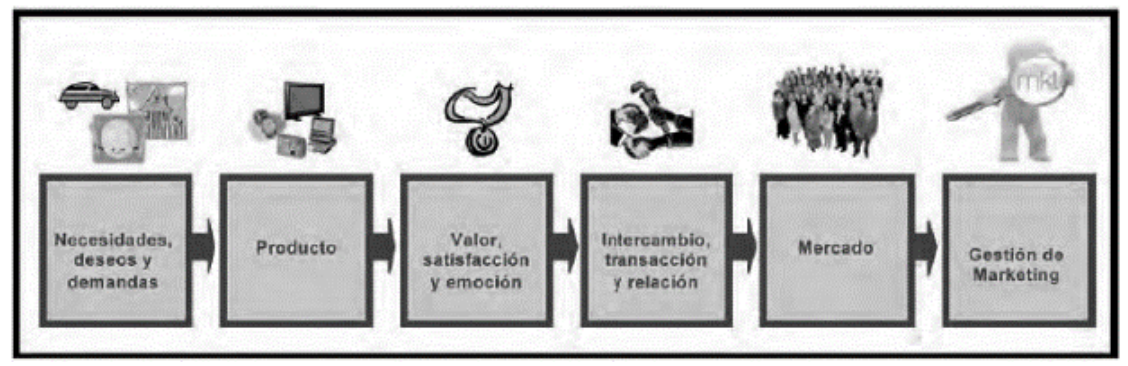

Fig 3. Definición de marketing de la AMA. Fuente: (Monferrer, 2013) 
El desarrollo del marketing a impulsado el desarrollo de las organizaciones y esto a su vez impulsa el desarrollo de las sociedades al incrementarse las tasas de trabajo. El desarrollo económico y social alcanza sus cuotas más altas en los países en los que sea mayor la preponderancia a la innovación y uso del marketing (Muñiz, 2018)

El concepto de marketing es considerado total solo cuando los aspectos relacionados con las implicaciones que la aplicación del marketing genera en la sociedad (aspectos tecnológicos y ecológicos) entren a formar parte de una definición de marketing (Munuera, 1992)

Para que tenga esa connotada definición de concepto total se debe involucrar el marketing al medio ambiente. Es involucrar a este término a la filosofía de la empresa. No es sólo asumir la responsabilidad social mínima, sino ayudar a la mejora del sistema ecológico dentro del que se encuentra el sistema empresarial (Calomarde, 2000).

Es por ello, que le marketing ecológico se ubica en el ámbito del marketing social corporativo e integra los objetivos sociales con los del marketing comercial empresarial en lo que respecta a la protección ambiental (Díaz, 2008).

Estos mismos estudios de marketing ecológico deben ser aplicado a los productos ecológicos. Lo que si se debe aplicar es romper las barreras de mercadeo para poder impulsar estos productos. Este reto pasa necesariamente por hacer totalmente asequible el producto al mercado, es decir ofrecer el producto con un precio y un aspecto visual lo más similar posible al producto convencional y, si es posible, en los mismos puntos de venta que éste (Rivera \& Sánchez, 2002).

Pero esto no afectará aquellos consumidores de conciencia ecológica ya que están dispuestos a hacer esfuerzos importantes por lo cual no tendrán problema en buscar un producto fuera de los canales masivos de comercialización (Izagirre, Fernández, \& Vicente, 2013). Esto se puede detallar con más ímpetu en la Fig. 4.

Por lo tanto, esta investigación tiene como objetivo general analizar la fabricación de cajas de cartón con material reciclado a través del marketing ecológico a nivel empresarial. La metodología utilizada se basó en investigación exploratoria descriptiva tomando como fundamentos las definiciones del reciclado, fabricación con materiales reciclados, marketing, marketing ecológico y producto ecológico. 


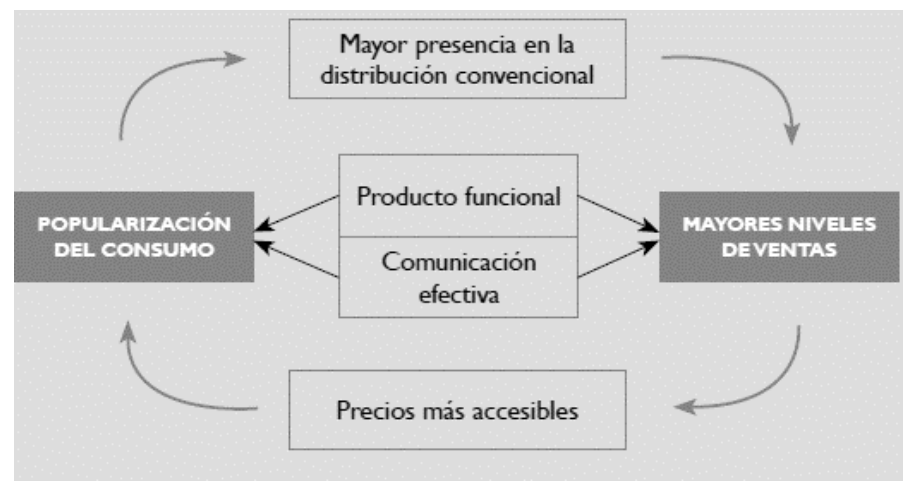

Fig 4. Circulo virtuoso de la comercialización de productos ecológicos. Fuente: (Izagirre, Fernández, \& Vicente, 2013).

\section{Método}

El diseño de esta investigación es no experimental, de campo documental y exploratoria descriptiva. Esto es debido a que se recopila datos de forma directa con los consumidores y empresas. Esta información se basa directamente en comprender la terminología de reciclado, fabricación con materiales reciclados, marketing, marketing ecológico y producto ecológico. La metodología se basó en técnicas basadas en revisión de textos, documentos y artículos científicos publicados disponibles en la web.

\section{Funcionamiento del marketing ecológico en el sector empresarial}

En el esquema ecológico, el marketing debe generar un aporte en el desarrollo de la compañía, de tal manera que plantee estrategias comerciales que permitan satisfacer las necesidades del cliente. Para esto el Marketing ecológico debe tomar como misión 3 funciones: (1) Redirigir la elección de los consumidores, (2) Reorientar el marketing de la compañía, (3) Reestructurar el comportamiento de la empresa.

Según Calomarde (2000) existen instrumentos de marketing ecológico que están basados en:

Productos: Minimización de la contaminación y producción, sustitución de materiales escasos por abundantes, servicio al cliente de asesoramiento en uso ecológico y fabricación de productos reciclables y ahorradores de energía.

Precio: Discriminación positiva de precios, internalización de costes por contaminación y escasez de productos y diferenciación de precios ecológicos. 
Promoción / Comunicación: Concienciación ecológica, información sobre productos y fabricaciones ecológicas, promoción con criterios medioambientales, información sobre servicios ecológicos de los productos y realización de acciones de relaciones públicas sobre ecología.

Distribución: Fomento de la redistribución (Devolución y reciclado de envases, canales de distribución que ahorren en el consumo de recursos naturales y sensibilización ecológica en el punto de venta.

\section{Aplicación del marketing ecológico dentro de una organización empresarial}

Estrategia 1: Proponer a la empresa el cambio de logo con el fin de proyectar una imagen ecológica.

El logo de la propuesta, se diseñó de una manera ecologista, resaltando una caja de cartón corrugado, que protege el planeta y simula un empaque el cual protege el contenido que lleva dentro. Esto se ilustra en la Fig. 5.

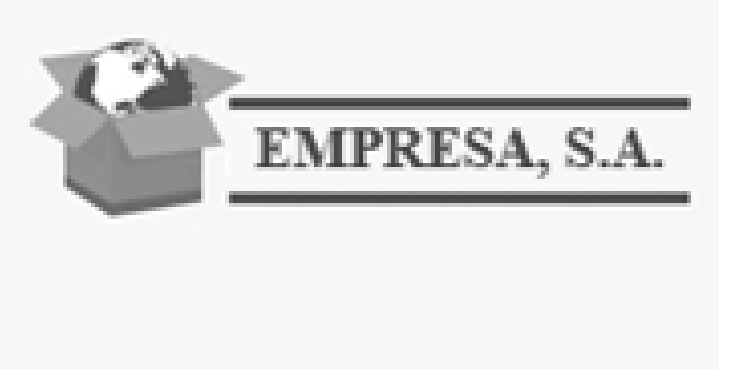

Fig 5. Logo de la empresa.

En cuanto a lo verbal se mantendrá el formato formal, precisamente para proyectar seriedad.

Las letras son de color verde con lo cual se relaciona con vida y renovación, un color que transmite tranquilidad y relajación, se asocia con la naturaleza y su vegetación.

La caja de cartón es de color café que evoca naturaleza, la tierra, expresa resistencia, defensa y confianza.

Estrategia 2: Redireccionar, el comportamiento de los empleados, a través de una campaña ecologista. 
Si se ofrece un producto ecológico, es de suma importancia iniciar con el cambio desde la compañía y sus empleados, para esto se plantean las siguientes actividades:

En cada lugar de trabajo el empleado deberá tener una planta natural, la cual deberá ser cuidada, para de esta manera inculcar la responsabilidad con el medio ambiente. Esto se detalla en la Fig. 6.

Se realizará actividades recreacionales una vez al mes durante 3 meses tales como: Sembrar plantas alrededor de la compañía, para así proyectar una imagen ecologista y fomentar a nuestros empleados a realizar minga de limpieza en los alrededores de la compañía.

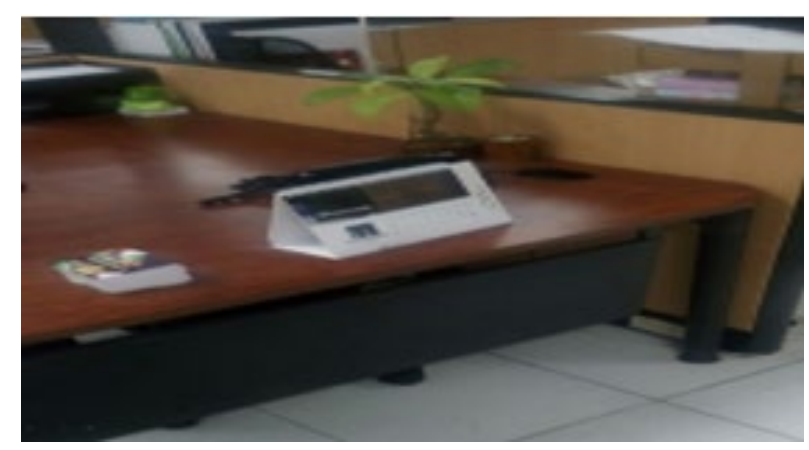

Fig 6. Lugar de trabajo con planta natural.

En cada área de la empresa, se aplicará publicidad ecológica que les recuerde a los empleados la nueva cultura ecologista en la que se está incursionando como: Rótulos en cada pasillo de la empresa para recordarles a los empleados la imagen ecologista de la empresa, como se observa en la Fig. 7, y Vinilos publicitarios en las puertas con el fin de decorar la entrada a cada área de la empresa, como se muestra en la Fig. 8.

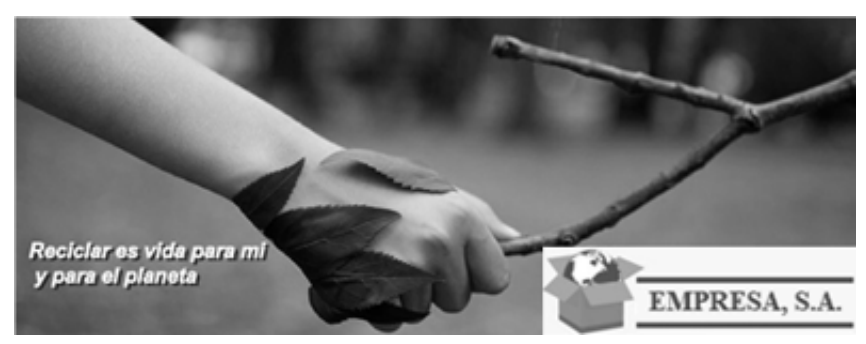

Fig 7. Rótulos con publicidad ecológica. 


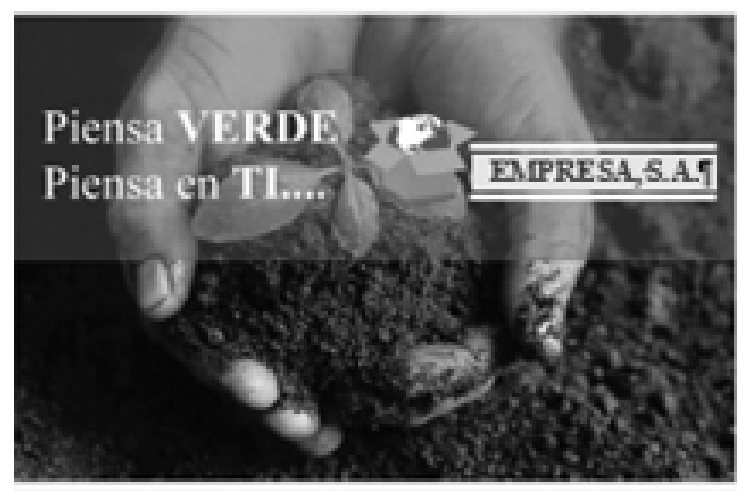

Fig 8. Vinilo con publicidad ecológica.

Realizar campañas de reciclaje dentro de la empresa, con los empleados aplicando las 3 R. Reduce, Reutiliza, Recicla. En la Fig. 9. se observa una publicidad sobre la campaña de reciclaje.

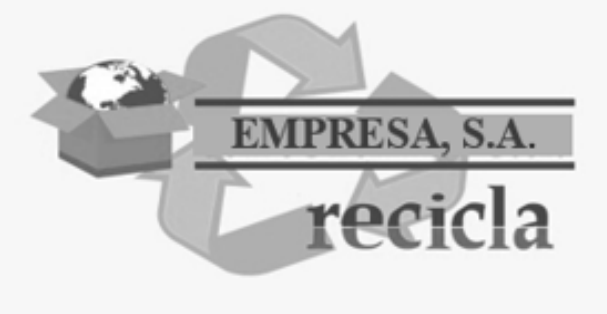

Fig 9. Publicidad sobre campaña de reciclaje dentro de la empresa.

Se ubicarán 4 tipos de contenedores de basura

Amarillo, destinado para productos plásticos y de metal.

Azul, destinado para papel y cajas de cartón, con el fin de reutilizarlo en el proceso productivo y así los empleados formaran parte de este proceso.

Verde. material de vidrio.

Gris. destinado para materia orgánica, plantas y compuestos biodegradables.

Estrategia 3: Estrategia de precios y reducción de costos del cliente.

Actualmente el dólar kilo de producto terminado se encuentra en $\$ 1.20$, es decir que por cada kilo de cajas vendidas una empresa cobra dicho valor, con el nuevo empaque ecológico se pretende disminuir este valor a $\$ 1.15$, si bien es cierto a simple vista la reducción de costo parece insignificante, pero las compras se miden por el volumen y es ahí donde se ve esta gratificante reducción en donde los clientes se verán beneficiados, debido a que los costos que le genera la 
compra de cajas de cartón para su proceso disminuirán, y ese valor que se ahorran pueden invertirlo en su negocio.

Se debe tomar en cuenta, que los clientes pueden tener la percepción de que "lo barato, sale caro", es decir pueden llegar a pensar que por ser un producto ecológico y de bajo precio este no le va a satisfacer las necesidades de su proceso, pero para esto se desarrolló la estrategia $\mathrm{N}^{\circ} 4$ presentada a continuación.

Estrategia 4: Demostración de producto ecológico.

Redirigir la forma de consumo de los clientes del mercado doméstico de la empresa es el reto de esta propuesta, es decir conseguir que los clientes consuman de una manera diferente.

\section{Para esto se plantea las siguientes actividades:}

Visitas técnicas a las instalaciones de planta de los clientes, para desarrollar pruebas industriales, que avalen el funcionamiento del empaque.

Capacitación a los clientes, sobre el uso correcto del empaque, esto debido a que varios clientes le dan un trato rustico a la caja lo que hace que esta pierda sus propiedades y por ende no cumpla con sus funciones la cual es proteger el producto que lleva dentro.

Programar visitas de los clientes a la planta industrial de la empresa, para que conozcan el proceso de producción de sus empaques. Esto para que los clientes se sientan parte de este cambio.

Promover el consumo responsable a través de charlas, dadas por ambientalistas y expertos del área de cartón.

Estrategia 5: Distribución Ecológica del producto.

Minimizar la generación de residuos durante la transportación, almacenamiento y manipulación, para esto se mantendrá relaciones comerciales con proveedores de transportes los cuales deben tener sus vehículos en buen estado, que no emanan gases tóxicos que pueden ser dañinos para el medio ambiente. 
Determinar un sistema de distribución inversa que permita el reciclaje con los clientes, es decir que cuando se les distribuya sus empaques de cartón para su proceso, estos envíen en el mismo camión aquellos empaques que ya han sido usados o están en desuso, para generar así de esta manera una cultura de reciclaje el cual servirá nuevamente como materia prima para futuras producciones.

La relación costo-beneficio para la implementación del marketing ecológico dentro de la organización.

Los costos para la realización de la propuesta de marketing ecológico para la empresa tienen un significado importante al principio para ser implantado durante un tiempo determinado. Estos valores son mínimos para el beneficio que tendrá en el ecosistema. Este marketing ecológico debe plantear el diseño de la campaña ecológica interna y externa de la compañía, la misma que servirá para redireccionar al empleado y al cliente a la nueva cultura ecológica a implementar.

Adicional con los empleados de la compañía se puede realizar actividades para inculcar el cuidado del medio ambiente aplicando las $3 \mathrm{R}$.

En cuanto a los clientes externos, se puede plantear realizar una demostración del producto guiado por expertos en el tema de cartón y expertos ambientales que avalen el correcto funcionamiento del empaque.

La implementación de esta propuesta es reducir el impacto ambiental que genera el consumo de papel importado en la fabricación de cajas de cartón, de esta manera se genera beneficio para la compañía, el cliente y el medio ambiente.

La empresa podrá reducir sus costos, de producción en un $16 \%$ aproximadamente, debido que, al comenzar a producir cajas de cartón con fibra reciclada para sus clientes domésticos.

Análisis de las propiedades del cartón reciclado para poder implementar las normas de calidad en el proceso de fabricación.

Para poder aplicar las normas de calidad internacional es necesario poder determinar las propiedades del papel reciclado. Un aspecto importante es la humedad en la fabricación cartón reciclado. La presencia de altos niveles de humedad ambiental, independientemente de la 
temperatura, disminuye las propiedades de resistencia de los papeles para la fabricación de cartón corrugado reciclado (Carracedo \& Gárate, 2005).

Para la fabricación de cartón reciclado se tiene una proporción general de cartón $60 \%$, periódico $25 \%$ y bond $15 \%$. La temperatura de la pulpa afecta la velocidad de refino e influye en la calidad del papel; al ser más baja la temperatura, más fácilmente se refina la pasta y el refino aumenta la resistencia a la tracción (Reyna, Robles, Toyohama, \& Canales). También Reyna at el, comentan que el batido de la pulpa, se debe regular la concentración, ya que una pulpa muy fluida retarda el batido y con una pulpa muy condensada se incrementa el gasto de energía.

Otro aspecto a considerar es la hidrofobidad del cartón reciclado. Para evitar esto se plantea una diversidad de resinas que se colocaran sobre cartones reciclados. Se utiliza una fórmula de $27 \%$ en peso de poliestireno expandido en un disolvente de limoneno, el cual demostraron una absorción del agua de dos minutos (Carrillo \& Gamboa, 2014).

Con estos resultados se puede implementar las normas internacionales de calidad, lo cual podrá permitir reducir los tiempos de entrega a través de la modificación de los estándares de calidad logrando así reducir las devaluaciones de materiales, evitar mermas innecesarias, reducir costos y controlar mejor los procesos a través de un Sistema de Gestión de Calidad (Mentado, 2015).

\section{Discusión}

Hoy en día las empresas importan una gran cantidad de papel de fibra virgen al año lo que está ocasionando que sus costos de producción se eleven debido al incremento del precio de la materia prima, a causa de la deforestación indiscriminada que existe en el entorno natural, ocasionando así un impacto ambiental negativo.

La mayoría de las empresas tienen procesos de producción agresivos con lo cual a través de estrategias de marketing ecológico se genera conciencia en los clientes en el uso de cajas de cartón con materia prima reciclada, sin afectar la calidad y características del producto.

Por lo tanto, con la implementación de las estrategias de marketing ecológico dará una mejora en la optimización de los recursos utilizados, además de minimizar los costos de producción en las empresas, como también lo hará el consumidor en los cuales transformará la forma de consumo y tratará de involucrarse en el tema ambiental al dejar de usar cajas de cartón con fibra virgen importada, a cajas con material reciclado, asimismo se ayudara a la preservación del medio 
ambiente ya que con la elaboración de empaques ecológicos conseguirá que se disminuya en algo la desforestación de los bosques.

\section{Referencias Bibliograficas}

Breiting, S. (1997). Hacia un nuevo concepto de educación ambiental. Carpeta informativa del CENEAM, 1-8.

Corraliza, J., \& Martín, R. (2000). Estilos de vida, actitudes y comportamientos ambientales. Medio Ambiente y Comportamiento Humano, 31-56.

Lara, J. (2008). Reducir, reutilizar y reciclar. Elementos 69, 45-48.

Henao, D. (2013). RECOLECCIÓN, MANEJO Y DISPOSICIÓN FINAL DE PAPEL RESIDUAL Y CARTÓN. Bogotá - Colombia: DESARROLLO ORGANIZACIONAL UNIVERSIDAD NACIONAL DE COLOMBIA.

Chacón, M., Pacheco, A., Cendejas, M., \& Ortega, F. (2016). Tendencia del crecimiento en la cultura del reciclaje. Revista de Ciencias Ambientales y Recursos Naturales, 63-72.

Castro, J. (2009). Dearrollar la cultura del reciclaje: Tarea de todos. Revista Digital Innovación y Experiencias Educativas, 1-10.

Chas, M., \& Lorenzo, M. (1997). EL RECICLAJE DE PAPEL Y CARTÓN EN LA UNIÓN EUROPEA. In Congresos Forestales, 147-152.

Aguilar, N. (2004). El reciclado de papel y cartón. Elementos 53, 54-56.

Cadme, N., \& Miranda, L. (2012). Estudio de factibilidad de una planta procesadora de papel y cartón reciclado en la ciudad de Azogues. Cuenca - Ecuador: Trabajo de Grado UNIVERSIDAD POLITÉCNICA SALESIANA.

CIFUENTES, J. (2013). ESTUDIO DE MERCADOS Y TÉCNICO PARA UNA EMPRESA DE FABRICACIÓN DE ENCUADERNADOS CON EMPAQUES RECICLADOS. Bogotá Colombia: Trabajo de Grado - UNIVERSIDAD DISTRITAL FRANCISCO JOSÉ DE CALDAS. Espinal, L. (2016). ANÁLISIS DEL PAPEL Y CARTÓN PROCEDENTES DEL RECICLAJE PARA POSIBLE APLICACIÓN EN EDIFICACIONES DE REPÚBLICA DOMINICANA. Barcelona - España: Trabajo de Grado - UNIVERSITAT POLITECNICA DE CATALUNYA. Monferrer, D. (2013). Fundamentos de marketing. Barcelona - España: Publicacions de la Universitat Jaume I. 
Muñiz, R. (26 de Septiembre de 2018). Marketing del siglo XXI. Obtenido de http://roa.ult.edu.cu/bitstream/123456789/2172/1/1.doc

Munuera, J. (1992). Evolución en las dimensiones del concepto de Marketing. ICE Revista de Economía, 126-142.

Calomarde, J. (2000). Marketing Ecológico. Madrid - España: Ed. Pirámide ESIC.

Dias, R. (2008). Marketing Ecológico y Turismo. Estudios y Perspectivas en Turismo, 140-155.

Rivera, L., \& Sánchez, M. (2002). Marketing de Productos Ecológicos. Mediterráneo Económico, 159-176.

Izagirre, J., Fernández, A., \& Vicente, M. (2013). Antecedentes y barreras a la compra de productos ecológicos. UNIVERSIA BUSINESS REVIEW, 108-127.

Carracedo, M., \& Gárate, B. (2005). Estudio de la resistencia de los papeles virgenes versus los papeles reciclados en la fabricación de cartón corrugado. Revista Iberoamericana de Ingeniería Mecánica, 119-124.

Reyna, L., Robles, R., Toyohama, L., \& Canales, V. (s.f.). Elaboración de cartón a partir de papel reciclado. Revista Peruana de Química e Ingeniería Química, 50-54.

Carrillo, J. G., \& Gamboa, R. (2014). Caracterización físico-mecánica de un cartón reciclado formulado para aplicaciones en contenedores de plantas. Mérida, Yucatán, México: Centro de Investigación Científica de Yucatán.

Mentado, I. (2015). Propuesta para la certificación del Sistema de Gestión de la Calidad (SGC) para el laboratorio de pruebas de una planta de fabricación de empaques de cartón corrugado. México, D. F. - México: Tesis de Grado - INSTITUTO POLITÉCNICO NACIONAL. 